\title{
Evaluación de la costo-efectividad de un modelo integral de tratamiento ambulatorio en pacientes con síndrome coronario agudo: aplicación de un modelo de Markov probabilístico
}

\author{
Kelly Salgado', Juan Carlos Salazar-Uribe², Jaime Gallo-Villegas ${ }^{3}$, Ángela Valencia4, \\ Diego Espíndola-Fernández ${ }^{4}$, Cristina Mesa ${ }^{4}$, Juan de la Calle ${ }^{1}$, Yanett Montoya1, \\ Dagnóvar Aristizába/4
}

Forma de citar

Salgado K, Salazar-Uribe JC, Gallo-Villegas J, Valencia A, Espíndola-Fernández D, Mesa C, et al Evaluación de la costo-efectividad de un modelo integral de tratamiento ambulatorio en pacientes con síndrome coronario agudo: aplicación de un modelo de Markov probabilístico. Rev Panam Salud Publica. 2018;42:e10. https://doi.org/10.26633/RPSP.2018.10

RESUMEN Objetivo. Evaluar la costo-efectividad de un modelo integral de tratamiento ambulatorio en pacientes que presentaron un sindrome coronario agudo.

Métodos. Se realizó una evaluación económica a partir de un estudio de intervención cuasi-experimental, que incluyó 442 pacientes con edades entre 30 y 70 años, quienes presentaron un síndrome coronario agudo. El grupo de intervención $(n=165)$ recibió un modelo integral de tratamiento ambulatorio fundamentado en el cuidado gestionado (disease management), mientras que el grupo control $(n=277)$ recibió rehabilitación cardiovascular convencional. Durante un año de seguimiento, se evaluó la presentación de reeventos cardiovasculares y hospitalizaciones. Se desarrolló un modelo de Markov probabilístico. La perspectiva de estudio se aplicó dentro del Sistema General de Seguridad Social en Salud Colombiano, incluidos los costos sanitarios directos; el horizonte temporal fue de 50 años con descuentos de 3,42\% para costos y efectividades; y la medida de efectividad fue los años de vida ganados ajustados por calidad. Se realizó un análisis de sensibilidad probabilístico y multivariante mediante de la simulación de Montecarlo.

Resultados. Durante el año de seguimiento, los costos directos relacionados con el valor pagado fueron en promedio de USD 2577 para el grupo control y USD 245 para el grupo de intervención. En el análisis de sensibilidad probabilístico, 91,3\% de las simulaciones se ubicaron en el cuadrante correspondiente a costos incrementales negativos y efectividades incrementales positivas (intervención evaluada de menor costo, más efectiva). En las simulaciones, se observó un ahorro promedio anual por paciente de USD 1215 por cada año de vida ganado ajustado por calidad.

Conclusiones. El modelo integral de tratamiento ambulatorio implementado en pacientes que sufrieron un síndrome coronario agudo mostró ser menos costoso y más efectivo en comparación con el cuidado convencional. Por ser una alternativa dominante, se recomienda como modelo de cuidado en esta población.

Palabras clave Evaluación de costo-efectividad; cadenas de Markov; síndrome coronario agudo; cuidado ambulatorio; cuidado gestionado; rehabilitación cardíaca; Colombia.

Entidad Promotora de Salud SURA, Medellín, Colombia.

2 Escuela de Estadística, Universidad Nacional de Colombia, Sede Medellín, Colombia.
Facultad de Medicina, Universidad de Antioquia, Medellín, Colombia.

4 Centro Clínico y de Investigación Soluciones Integrales de Conocimiento en Riesgo Cardio- vascular, Medellín, Colombia. Enviar la correspondencia a Dagnóvar Aristizábal: dagnovar@une.net.co

Este es un artículo de acceso abierto distribuido bajo los términos de la licencia Creative Commons Attribution-NonCommercial-NoDerivs 3.0 IGO, que permite su uso, distribución y reproducción en cualquier medio, siempre que el trabajo original se cite de la manera adecuada. No se permiten modificaciones a los artículos ni su uso comercial. Al reproducir un artículo no debe haber ningún indicio de que la OPS o el artículo avalan a una organización un producto específico. El uso del logo de la OPS no está permitido. Esta leyenda debe conservarse, junto con la URL original del artículo. 
La enfermedad coronaria es un problema de salud pública en países desarrollados y emergentes debido a su alta morbilidad y mortalidad (1). Luego de un síndrome coronario agudo (SCA), la rehospitalización es una situación frecuente que puede necesitar reintervenciones que prolongan la estancia hospitalaria y mayores demandas de recursos al sistema de salud (2).

En los países desarrollados, el cuidado transicional y los modelos integrales de tratamiento ambulatorio (MITA) fundamentados en el cuidado gestionado (disease management) han permitido la integración de redes de prestación de servicios de salud y un mejor cuidado (3-6), con mayor eficiencia, disminución en el número de hospitalizaciones, eventos prevenibles y reducción en costos de atención $(3,4,7,8)$.

En el contexto sociocultural latinoamericano se desconoce la costo-efectividad de los MITA en pacientes luego de un SCA. Debido al aumento de los gastos en salud, los entes sanitarios y los sistemas de aseguramiento demandan la realización de estudios de evaluación económica de tecnologías sanitarias para contribuir al desarrollo de mejores políticas públicas, estructurar los programas de salud y tomar las mejores decisiones basadas en la evidencia científica.

En la actualidad, los modelos de Markov representan uno de los métodos de valoración científica más utilizados para la evaluación económica de tecnologías sanitarias (9). Si bien este tipo de modelos tienen limitaciones metodológicas, permiten simular el curso de enfermedades crónicas, enfermedades con ciclos repetitivos y enfermedades irreversibles a través del tiempo, a partir de representaciones matemáticas de componentes clínicos y epidemiológicos de una forma rigurosa y de fácil comprensión $(9,10)$.

Recientemente, nuestro grupo reportó una disminución de $40 \%$ en las consultas al servicio de urgencias y rehospitalizaciones por nuevos eventos cardiovasculares en pacientes con SCA atendidos en un MITA (11). Sin embargo, se desconoce la costo-efectividad del modelo de atención ambulatorio implementado. Por tal razón, el objetivo de este estudio fue evaluar la costo-efectividad de un MITA en pacientes que presentaron un SCA.

\section{MATERIALES Y MÉTODOS}

Se realizó un estudio de intervención cuasiexperimental, que evaluó la costoefectividad de un MITA en pacientes que presentaron un SCA y que incluyó dos grupos: un grupo de intervención conformado por 165 pacientes, atendidos en un MITA en la institución Centro Clínico y de Investigación Soluciones Integrales de Conocimiento en Riesgo Cardiovascular (SICOR), Medellín (Colombia) entre 2010 y 2011; y un grupo control, conformado por 277 pacientes atendidos entre 2008 y 2009 (cohorte histórica). Ambos grupos estaban afiliados a la Entidad Promotora de Salud SURA (EPS SURA) y egresaron luego de presentar un SCA que, en general, requirió una angiografía coronaria con revascularización percutánea o quirúrgica (11).

Se realizó el seguimiento de cada individuo durante un año para evaluar la presentación de nuevos eventos cardiovasculares que requirieron la visita al servicio de urgencias $u$ hospitalización. A partir de la historia clínica electrónica se obtuvo información del evento coronario agudo (evento índice). La medición del desenlace la realizó un funcionario independiente del grupo de investigadores, que desconocía a qué grupo pertenecía el paciente.

Se incluyeron personas entre 30 y 70 años, con diagnóstico de SCA, con o sin elevación del segmento ST en el trazado electrocardiográfico, o episodios de angina que requirieron una hospitalización. Se eligió un rango de edad donde los costos inherentes a la atención fueran directos de la enfermedad coronaria y no debidos a otras comorbilidades que surgen con la edad, o fueran derivados de una cardiopatía secundaria avanzada como la insuficiencia cardíaca. Se excluyeron pacientes embarazadas, pacientes con creatinina mayor o igual a $2 \mathrm{mg} / \mathrm{dL}$, nefropatía diabética con proteinuria manifiesta mayor o igual a un gramo en 24 horas, cor pulmonar asociado, trastorno cognitivo o ausencia de un cuidador primario.

\section{Intervención}

El grupo de intervención recibió un MITA con cuidado transicional (6) y cuidado gestionado, que incluyó la atención por un grupo interdisciplinario conformado por un cardiólogo, un especialista en medicina del ejercicio, un médico general, un fisioterapeuta y una enfermera. La enfermera coordinó el plan de cuidado inicial posthospitalario (PCPH) el cual contenía los diagnósticos, información de contacto del cuidador primario, fechas de las citas, medicamentos, exámenes diagnósticos pendientes e información sobre manejo de síntomas. Se realizó un contacto posegreso y acompañamiento telefónico (12).

El seguimiento ambulatorio incluyó evaluación clínica, ayudas diagnósticas según comorbilidades y gravedad, telemetría fisiológica, acompañamiento telefónico para síntomas, adherencia al plan terapéutico, soporte emocional y educación para el autocuidado $(3,13)$. Se utilizó la cardiografía de impedancia para la detección temprana de descompensación (falla cardíaca) o ajuste farmacológico $(14,15)$ y un plan de tratamiento coordinado por un cardiólogo mediante el uso de guías basadas en evidencia $(16,17)$. Durante la intervención, la enfermera coordinó, vía telefónica, el acceso de los pacientes a una atención médica inmediata o en las 24 horas siguientes a la aparición de algún síntoma.

El grupo control recibió el tratamiento convencional con rehabilitación cardíaca y consultas ambulatorias por cardiología y medicina general, pero sin cuidado gestionado.

\section{Aspectos éticos}

El estudio fue aprobado por el Comité de Ética de la EPS SURA. Se obtuvo el consentimiento informado de cada individuo que ingresó al MITA. Se tuvieron en cuenta las normas para la investigación en salud del Ministerio de la Protección Social de Colombia, Resolución 008430 de 1993 (18) y los principios de la Declaración de Helsinki (19).

\section{Análisis estadístico}

Se utilizó la prueba de Shapiro-Wilks para evaluar si las variables provenían de una población con distribución normal. Para la descripción de las variables cuantitativas se utilizaron el promedio, la desviación estándar, la mediana y el rango intercuartílico, según el tipo de distribución. Para la descripción de las variables nominales se utilizaron proporciones. 
La comparación entre los grupos de tratamiento en las variables cuantitativas se realizó con la prueba t de Student, a excepción de la variable días de hospitalización evento índice en la cual se utilizó la prueba de U de Mann-Whitney debido al no cumplimiento del supuesto de distribución normal; en las variables cualitativas, se utilizó la prueba chi cuadrado de independencia. Para la estimación del efecto de la intervención, se utilizó un análisis multivariado de riesgos proporcionales de Cox ajustado por potenciales variables de confusión.

La evaluación económica de la tecnología sanitaria implementada incluyó las siguientes fases:

- construcción del modelo de Markov,

- ajuste del modelo de regresión logística multinomial por edad y sexo,

- cálculo de la matriz de probabilidades de transición para el modelo de Markov de tres estados ajustado por el modelo de regresión logística multinomial,

- simulación de la matriz de permanencia de los pacientes a lo largo del tiempo para los dos grupos de tratamiento,

- simulación de los costos, efectividades, costos incrementales y efectividades incrementales y

- generación del gráfico con el plano de costo-efectividad.

Se desarrolló un modelo de Markov de tres estadios (paciente en riesgo, evento cardiovascular y muerte), con el fin de realizar la evaluación económica de las dos alternativas de tratamiento, con las probabilidades de transición de estados luego de un SCA. Debido a las diferencias en la incidencia de eventos cardiovasculares según la edad y el sexo (1), se utilizó un modelo de regresión logística multinomial, con ajuste de probabilidades de transición por estas dos covariables (20).

Para la evaluación económica, los pacientes fueron seguidos hasta el final de sus vidas con un horizonte temporal de 50 años a partir de la intervención. La duración de cada ciclo del modelo correspondió a un año de vida, debido a que esta fue la duración del tratamiento. La perspectiva del estudio fue la del Sistema General de Seguridad Social en Salud de Colombia (SGSSS) e incluye costos sanitarios directos relacionados con el valor pagado por una aseguradora por

las prestaciones (cuidado ambulatorio, intervenciones quirúrgicas, hospitalizaciones, entre otras). Se consideraron los costos de todas las prestaciones de salud con las mismas tarifas para los dos grupos de tratamiento.

La estimación de los costos asociados a cada una de las alternativas se hizo a partir de un ajuste distribucional estadístico de los costos reales. La medida de efectividad fueron los años de vida ganados ajustados por calidad (AVAC). Dado que no se disponía de una medición de la calidad de vida en el grupo control por ser una cohorte histórica, las utilidades de cada alternativa en términos de ganancia, según lo establecido por la aplicación del instrumento de medición de la salud EQ-5D (21), fueron tomadas de un estudio previo de otros autores donde se calcularon y establecieron los valores de utilidad para pacientes en un programa de promoción de la salud y prevención cardiovascular (22). Se consideró una utilidad del estado evento de 0,841 , del estado riesgo de 1 y del estado muerte de 0 (22).

La menor valoración del costo y de la efectividad futuros se corrigió mediante un descuento en ambos conceptos de $3,42 \%$ de acuerdo con el promedio del índice de precios al consumidor (IPC) de salud en Colombia para los últimos 13 años.

Los resultados fueron expresados mediante la razón de costo-efectividad incremental (RCEI) (23), en la cual se analizan los costos y los efectos sobre la salud en términos comparativos mediante la siguiente fórmula:

\section{$R C E I=\frac{C I-C C}{E I-E C}=\frac{\text { costo de la intervencion }- \text { costo del control }}{\text { efectividad de la intervencion }- \text { efectividad del control }}$}

Como análisis de sensibilidad probabilística, se estimaron las probabilidades de transición a partir de modelos de regresión logística multinomial donde las covariables edad y sexo fueron introducidas como distribuciones ajustadas según los datos reales de los grupos de tratamiento y las tablas de vida por edad y sexo para personas colombianas (24). Para representar la incertidumbre en los resultados y su propagación desde los parámetros, se utilizó la simulación de Montecarlo. Las distribuciones de probabilidades utilizadas para estimar los parámetros del modelo de Markov fueron: distribución Log normal para costos de los estados riesgo y evento, distribución ß general para la edad y distribución binomial para sexo.

Por último, mediante una simulación estadística, se evaluó el comportamiento de 1000 pacientes en cada grupo. Se calculó el delta del costo promedio por paciente de 1000 grupos simulados y el delta del promedio de los AVAC por paciente de los mismos 1000 grupos simulados. El procesamiento del modelo de Markov se realizó según las guías actuales (25) multiplicando el vector fila, que indica la distribución inicial del grupo en los diferentes estados de salud, por la matriz de probabilidades de transición ajustadas con el modelo de regresión logística multinomial. Dicho proceso finalizó cuando todos los participantes alcanzaron el estado absorbente (muerte) luego de 50 ciclos. La efectividad de cada ciclo se obtuvo multiplicando la supervivencia por la utilidad del estado y el descuento de cada ciclo; luego, se sumó la efectividad de cada alternativa y se dividió por 1000 para obtener la efectividad promedio de cada paciente por grupo. El proceso de simulación y la obtención de los resultados de los modelos se obtuvo a partir del software @Risk.

Para el análisis descriptivo y bivariado se usó un nivel de significancia estadística $\alpha=0,05$ y se empleó el software IBM SPSS Statistics, versión 21.0.

\section{RESULTADOS}

Se incluyeron en el análisis 165 pacientes del grupo de intervención y 277 pacientes del grupo control, con un

promedio de edad de 58,3 \pm 7,6 años, de los cuales $70,2 \%$ eran hombres. No se observaron diferencias entre los dos grupos en la mayoría de las características descritas (cuadro 1). Se presentaron 150 eventos cardiovasculares, en promedio 0,41 en el grupo control y 0,22 en el grupo de intervención $(P=0,003)$. En el análisis de riesgos proporcionales de Cox, el riesgo de un evento cardiovascular fue mayor en el grupo control en comparación con el grupo de intervención (cociente de riesgo [CR]: 1,8; intervalo de confianza del 95\% [IC95\%] 1,17-2,75; $P=0,007$ ) luego de ajustar por la edad, sexo, antecedente de tabaquismo y arterias coronarias 
comprometidas; un hallazgo similar se obtuvo al tener en cuenta la realización de algún procedimiento de revascularización (CR: 2,0; IC95\%: 1,20-3,12; P = 0,007.

Durante el año de seguimiento, los costos directos relacionados con el valor pagado por la aseguradora fueron, en promedio, de USD 2577 para el grupo control y USD 2245 para el grupo de intervención. El valor pagado por la aseguradora en promedio por las prestaciones ambulatorias electivas, ambulatorias urgentes, hospitalarias electivas y hospitalarias urgentes, en el grupo control y en el grupo de intervención fue USD 852 versus USD 692; USD 9,0 versus USD 10,5; USD 300 versus USD 192 y USD 1415 versus USD 1349 , respectivamente. Con relación a la frecuencia de uso, la proporción de pacientes que requirió alguna prestación ambulatoria electiva, ambulatoria urgente, hospitalaria electiva y hospitalaria urgente en el grupo control y el grupo de intervención fue $96,4 \%$ versus $96,3 \%$, $10,6 \%$ versus $10,1 \%, 5,5 \%$ versus $4,3 \%$ y $30,7 \%$ versus $23,2 \%$, respectivamente.

CUADRO 1. Descripción de las características demográficas, clínicas y de laboratorio, según el grupo de tratamiento

\begin{tabular}{|c|c|c|c|}
\hline \multirow[b]{2}{*}{ Variable } & \multicolumn{2}{|c|}{ Grupo } & \multirow[b]{2}{*}{ Valor $P$} \\
\hline & $\begin{array}{c}\text { Control } \\
(n=277)\end{array}$ & $\begin{array}{l}\text { Intervención } \\
\quad(n=165)\end{array}$ & \\
\hline Edad (años) & $58,7 \pm 7,3$ & $57,7 \pm 8,2$ & $0,208^{a}$ \\
\hline Sexo masculino $(\%)$ & 71,7 & 67,7 & $0,368^{b}$ \\
\hline Antecedente de hipertensión arterial (\%) & 69,3 & 62,4 & $0,137^{b}$ \\
\hline Antecedente de diabetes mellitus (\%) & 20,2 & 18,2 & $0,601^{b}$ \\
\hline Antecedente de dislipidemia (\%) & 55,2 & 63,6 & $0,083^{b}$ \\
\hline Antecedente de tabaquismo (\%) & 28,5 & 46,7 & $<0,001^{\mathrm{b}}$ \\
\hline Antecedente de infarto de miocardio (\%) & 14,4 & 11,5 & $0,382^{b}$ \\
\hline Antecedente de angioplastia (\%) & 38,6 & 35,2 & $0,465^{b}$ \\
\hline Antecedente de bypass (\%) & 12,6 & 11,5 & $0,728^{b}$ \\
\hline Días de hospitalización evento índice (mediana p25-75) & $3,0(2,0-6,0)$ & $3,0(2,0-5,0)$ & $0,797^{c}$ \\
\hline Presión arterial sistólica (mmHg) & $136,4 \pm 24,3$ & $137,3 \pm 23,5$ & $0,827^{\mathrm{a}}$ \\
\hline Presión arterial diastólica (mmHg) & $78,1 \pm 13,6$ & $78,9 \pm 12,3$ & $0,733^{\mathrm{a}}$ \\
\hline Frecuencia cardíaca (latidos/min) & $72,4 \pm 13,7$ & $73,7 \pm 14,3$ & $0,611^{\mathrm{a}}$ \\
\hline Fracción de eyección del ventrículo izquierdo (\%) & $50,0 \pm 11,4$ & $47,8 \pm 10,3$ & $0,082^{\mathrm{a}}$ \\
\hline Colesterol total (mg/dL) & $184,3 \pm 45,7$ & $199,6 \pm 45,0$ & $0,061^{\mathrm{a}}$ \\
\hline Triglicéridos (mg/dL) & $220,8 \pm 194,9$ & $220,8 \pm 110,1$ & $0,999^{a}$ \\
\hline Colesterol HDL (mg/dL) & $37,1 \pm 8,7$ & $38,1 \pm 10,4$ & $0,590^{\mathrm{a}}$ \\
\hline Requirió coronariografía (\%) & 85,9 & 91,5 & $0,080^{\mathrm{b}}$ \\
\hline Requirió angioplastia primaria (\%) & 60,3 & 61,2 & $0,848^{b}$ \\
\hline Requirió bypass (\%) & 15,2 & 16,4 & $0,736^{b}$ \\
\hline Diagnóstico de angina inestable al egreso (\%) & 28,9 & 28,5 & $0,929^{b}$ \\
\hline Diagnóstico de IAM sin ST al egreso (\%) & 23,1 & 28,5 & $0,207^{b}$ \\
\hline Diagnóstico de IAM con ST al egreso (\%) & 22,0 & 19,4 & $0,512^{\mathrm{b}}$ \\
\hline Diagnóstico de falla cardíaca al egreso (\%) & 2,5 & 3,6 & $0,504^{b}$ \\
\hline
\end{tabular}

Cuadro de elaboración propia a partir de los resultados. Datos presentados como promedio \pm desviación estándar (DE) cuando es apropiado.

HDL, lipoproteína de alta densidad (por sus siglas en inglés); IAM, infarto agudo de miocardio; ST, segmento ST.

a Se utilizó la prueba t de Student.

' Se utilizó la prueba chi cuadrado de independencia.

' $S e$ utilizó la prueba U de Mann-Whitney.

En el cuadro 2 se presentan las probabilidades de transición del modelo de Markov de tres estados ajustado por el modelo de regresión logística multinomial; se incluyeron la edad y el sexo como covariables. La probabilidad de un nuevo evento cardiovascular fue de 30\% para el grupo control y $15 \%$ para el grupo de intervención.

En el cuadro 3 se muestran los resultados de la evolución de los grupos de tratamiento a lo largo de una muestra de 17 ciclos de un total de 50 ciclos, iniciando con 1000 pacientes en el estado riesgo, 0 pacientes en el estado evento y 0 pacientes en el estado muerte. La efectividad correspondiente a la medición de las utilidades de cada alternativa en términos de AVAC fue en promedio de 1,13 para el grupo de intervención y 0,76 para el grupo control.

El plano de costo-efectividad muestra que $97,3 \%$ de las simulaciones se ubican entre el cuadrante 2 y el 3, a predominio del cuadrante 2 con $91,3 \%$ del total de las simulaciones (figura 1). Esto indica que la aplicación del MITA luego de un SCA es menos costoso y más efectivo; por consiguiente, la alternativa se considera dominante.

Las simulaciones para la RCEI mostraron un ahorro en promedio de USD 60780366 a lo largo de 50 años para 1000 pacientes simulados, correspondiente a un total anual de USD 1215607 y un promedio anual por paciente de USD 1215 (figura 2).

\section{DISCUSIÓN}

Nuestros hallazgos muestran que el MITA implementado en pacientes que sufrieron un SCA es menos costoso y más efectivo. Se observó una RCEI en promedio anual por paciente de USD 1215 de ahorro por cada AVAC. Estos resultados permiten clasificar la intervención evaluada como dominante y se recomienda su adopción como estrategia de tratamiento. El horizonte temporal no

CUADRO 2. Probabilidades de transición utilizadas en el modelo de Markov de tres estados ajustadas por edad y sexo en un modelo de regresión logística multinomial para los dos grupos de tratamiento

\begin{tabular}{|c|c|c|c|c|c|c|}
\hline & \multicolumn{3}{|c|}{ Grupo de intervención } & \multicolumn{3}{|c|}{ Grupo control } \\
\hline & $\begin{array}{c}\text { Estado riesgo } \\
(\%)\end{array}$ & $\begin{array}{c}\text { Estado evento } \\
\text { (\%) }\end{array}$ & $\begin{array}{c}\text { Estado muerte } \\
(\%)\end{array}$ & $\begin{array}{c}\text { Estado riesgo } \\
(\%)\end{array}$ & $\begin{array}{c}\text { Estado evento } \\
(\%)\end{array}$ & $\begin{array}{c}\text { Estado muerte } \\
(\%)\end{array}$ \\
\hline Estado riesgo & 83,0 & 15,0 & 1,0 & 67,0 & 30,0 & 2,8 \\
\hline Estado muerte & 0,0 & 0,0 & 100,0 & 0 & 0 & 100,0 \\
\hline
\end{tabular}

Cuadro de elaboración propia a partir de los resultados presentados. 
CUADRO 3. Muestra de 17 ciclos de 50 del procesamiento del modelo de Markov a partir de 1000 individuos en riesgo en cada grupo de tratamiento

\begin{tabular}{|c|c|c|c|c|c|c|}
\hline \multirow[b]{2}{*}{ Ciclo } & \multicolumn{3}{|c|}{ Grupo de intervención } & \multicolumn{3}{|c|}{ Grupo control } \\
\hline & $\begin{array}{l}\text { Número de } \\
\text { personas en } \\
\text { riesgo }\end{array}$ & $\begin{array}{l}\text { Número de } \\
\text { eventos }\end{array}$ & $\begin{array}{l}\text { Número de } \\
\text { muertes }\end{array}$ & $\begin{array}{l}\text { Número de } \\
\text { personas en } \\
\text { riesgo }\end{array}$ & $\begin{array}{l}\text { Número de } \\
\text { eventos }\end{array}$ & $\begin{array}{l}\text { Número de } \\
\text { muertes }\end{array}$ \\
\hline Vector Inicial & 1000 & 0 & 0 & 1000 & 0 & 0 \\
\hline Año 1 & 820 & 170 & 10 & 734 & 239 & 27 \\
\hline Año 2 & 666 & 306 & 28 & 555 & 397 & 48 \\
\hline Año 3 & 556 & 400 & 44 & 414 & 512 & 74 \\
\hline Año 4 & 465 & 480 & 55 & 298 & 600 & 102 \\
\hline Año 5 & 385 & 542 & 73 & 231 & 640 & 129 \\
\hline Año 6 & 323 & 624 & 90 & 161 & 687 & 161 \\
\hline Año 7 & 269 & 645 & 107 & 111 & 690 & 199 \\
\hline Año 8 & 220 & 658 & 135 & 79 & 691 & 230 \\
\hline Año 9 & 187 & 683 & 155 & 65 & 675 & 260 \\
\hline Año 10 & 146 & 680 & 171 & 49 & 647 & 304 \\
\hline Año 11 & 121 & 684 & 199 & 35 & 626 & 339 \\
\hline Año 12 & 93 & 675 & 223 & 25 & 604 & 371 \\
\hline Año 13 & 71 & 655 & 254 & 19 & 578 & 403 \\
\hline Año 14 & 56 & 635 & 289 & 13 & 550 & 437 \\
\hline Año 15 & 48 & 616 & 317 & 8 & 508 & 484 \\
\hline Año 16 & 38 & 597 & 346 & 6 & 468 & 526 \\
\hline Aก̃o 17 & 28 & 569 & 375 & 3 & 439 & 558 \\
\hline
\end{tabular}

Cuadro elaborado por los autores a partir de los resultados presentados.

FIGURA 1. Plano de costo-efectividad que compara el modelo integral de tratamiento ambulatorio (grupo de intervención) con el cuidado convencional (grupo control) luego de un síndrome coronario agudo.



Mínimo: USD -6 286 dólares; máximo: USD 5 286; promedio: USD -60,8; DE: USD 471,4; número de simulaciones: 1 000. Se observa un ahorro por cada unidad de efectividad incremental de USD 60780366 en promedio a lo largo de 50 años para 1000 pacientes simulados.

AVAC, años de vida ganados ajustados por calidad. se escogió de manera deliberada, sino que corresponde a la evolución de las simulaciones realizadas en el modelo propuesto. En este modelo puede hacerse un seguimiento de los desenlaces hasta que el total de la población llegue al final de su vida, para observar la mayor cantidad de información en la cohorte.

Uno de los pilares de la intervención fue el cuidado gestionado, que consistió en la optimización de la atención sanitaria mediante la coordinación de recursos de forma integral para obtener resultados favorables $(3,13)$. Si bien algunos programas de rehabilitación cardíaca incluyen este concepto, la mayoría hace énfasis en cambios en el estilo de vida con el fin de controlar los factores de riesgo a través de consejería, educación, seguimiento clínico y prescripción del ejercicio (26). El cuidado gestionado incorporado dentro del MITA fue el principal componente diferenciador de nuestra estrategia de tratamiento y podría explicar los resultados observados. El MITA descrito se fundamentó en intervenciones ajustadas al nivel de gravedad y el seguimiento proactivo, para detectar descompensaciones en forma temprana mediante algoritmos clínicos (27), métodos diagnósticos que facilitan ajustes terapéuticos $(15,28,29)$ y la promoción de la salud basada en el autocuidado (30).

La mayoría de los estudios de evaluación económica han reportado que la rehabilitación cardíaca es una estrategia dominante de menor costo y mayor efectividad cuando se la compara con ningún tratamiento (31). Un estudio realizado en China reportó una RCEI de USD 640 en 2004 por cada AVAC cuando se la comparó un programa de rehabilitación cardíaca con ningún tratamiento (32). Asimismo, un programa de rehabilitación cardíaca frente a ninguna intervención en pacientes con falla cardíaca reportó una RCEI de USD 1773 en 2001 por cada AVAC (33).

Lo anterior se ha visto confrontado con los hallazgos de mayor costo y efectividad de los programas de rehabilitación cardíaca cuando se comparan con el cuidado convencional (34). Marchionni et al. reportaron que la rehabilitación cardíaca fue más costosa y más efectiva respecto al cuidado convencional en pacientes con antecedente de infarto de miocardio (35). Un hallazgo similar fue reportado en Canadá, con una RCEI de USD 9200 en 1993 por cada AVAC al comparar un programa de rehabilitación 
FIGURA 2. Distribución de las simulaciones de la razón costo-efectividad incremental que compara el modelo integral de tratamiento ambulatorio (grupo de intervención) con el cuidado convencional (grupo control) luego de un síndrome coronario agudo.

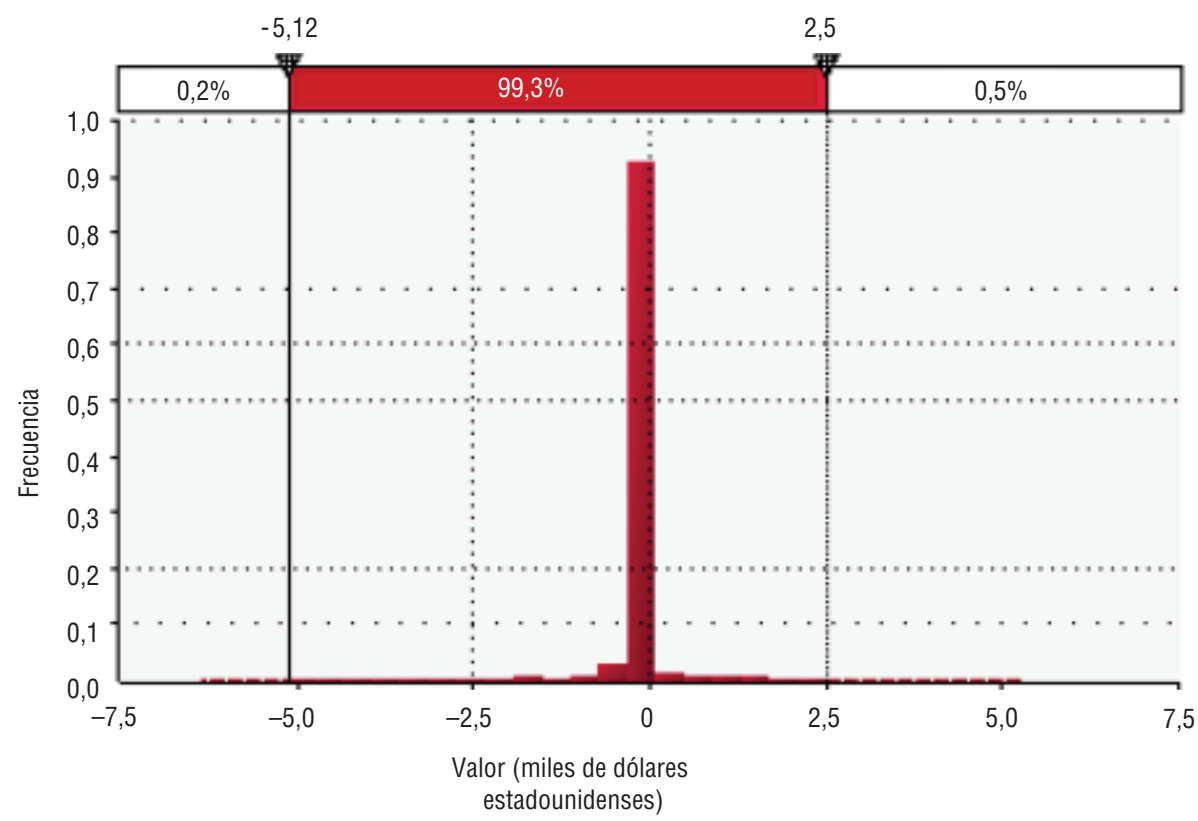

Promedio en $x$ : 0,36; DE en $x$ : 0,16; promedio en $y$ : USD -16,8 dólares; DE en y: USD 10,4; Coeficiente correlación de Pearson: 0,88. Cada punto representa el valor promedio de 1000 pacientes para 1000 grupos simulados.

cardíaca con el cuidado convencional (36). Estos resultados difieren con nuestros hallazgos y enfatizan la importancia de considerar la inclusión del cuidado gestionado en los programas de rehabilitación cardíaca.

Es importante resaltar que nuestro grupo control recibió el cuidado recomendado posterior al evento coronario $(17,37) \mathrm{y}$, por lo tanto, no se puede considerar un grupo sin tratamiento óptimo. Los pacientes del grupo control también ingresaron a rehabilitación cardíaca, lo que en principio haría más difícil mostrar alguna diferencia en la costo-efectividad entre los dos grupos de tratamiento. No obstante, los pacientes del grupo de intervención mostraron una menor frecuencia de prestaciones ambulatorias urgentes, hospitalarias ambulatorias y hospitalarias urgentes, lo cual se reflejó en los costos.

Las investigaciones que han medido la costo-efectividad de los MITA y programas de rehabilitación cardíaca luego de un SCA han reportado limitaciones metodológicas y alta variabilidad de resultados, lo que imposibilita tener una medida agregada (31). Entre las limitaciones se encuentran la ausencia de análisis de sensibilidad para evaluar la incertidumbre en costos y consecuencias, diferencias en los diseños, sistemas de salud, costos regionales $y$ tiempo de seguimiento, así como heterogeneidad en las intervenciones, tipos de pacientes y presunciones con relación a los costos (31). Sin embargo, los resultados de estos trabajos y el nuestro proveen evidencia que respaldan el desarrollo de nuevas prácticas alrededor del cuidado de los pacientes quienes han tenido un SCA.

Según el conocimiento de los autores, este es uno de los pocos estudios realizados en América Latina que evalúa la costo-efectividad de un MITA que incorpora estrategias de cuidado gestionado en el tratamiento de pacientes quienes presentaron un SCA. En los países de bajos y medianos ingresos las investigaciones son escasas y hay poca disponibilidad de los programas de rehabilitación cardíaca, a diferencia de lo que ocurre en los países desarrollados $(26,34)$. Por otra parte, se ha descrito la falta de estudios en América Latina en los que se hayan realizado evaluaciones económicas de programas de rehabilitación cardíaca $(26,38)$. Según la literatura indexada, solo se publicaron tres estudios de costo-efectividad, realizados en Brasil (39), Colombia (40) y Chile (41). En estas investigaciones, a diferencia de la nuestra, los programas de rehabilitación cardíaca basados en ejercicio fueron más costosos y más efectivos cuando se compararon con el cuidado convencional (26).

La limitación principal de esta investigación radica en que los resultados obtenidos provienen de un estudio cuasi-experimental; sin embargo, se hicieron los ajustes necesarios para controlar por potenciales variables de confusión (11). También, debido a que el grupo control correspondió a una cohorte histórica, se asumió una medida de utilidad de pacientes con características similares reportada por otros autores (22). Otro aspecto es el amplio rango de edad seleccionado como criterio de inclusión (entre 30 y 70 años). Sin embargo, en la muestra de estudio, $80 \%$ tenían entre 48 y 68 años. Por tal razón, la población representada estaba conformada en su mayoría por adultos de edad media y los resultados podrían ser extrapolables a personas que se hallan en esta etapa de la vida y son consistentes con los hallazgos epidemiológicos reportados en otros estudios (42). Además, los resultados del análisis de costo-efectividad se obtuvieron vía simulación basada en un modelo para enfermedad coronaria que incluyó proyecciones ajustadas para cada grupo de tratamiento, a partir de información de un estudio original. En este tipo de investigaciones, no es posible incorporara la atención de los pacientes los avances médicos y tecnológicos que van a ser desarrollados en el futuro. Recomendamos que las decisiones administrativas se fundamenten sobre todo en la valoración real de los costos asociados a cada grupo de tratamiento.

\section{CONCLUSIÓN}

El MITA implementado en pacientes que sufrieron un SCA con el fin de reducir la aparición de nuevos eventos cardiovasculares mostró ser menos costoso y más efectivo en comparación al cuidado convencional. Por tal razón, se clasifica como una alternativa dominante, se recomienda considerar su adopción como modelo de tratamiento e incluir el cuidado gestionado como un componen- 
te central en los programas de rehabilitación cardíaca.

Agradecimientos. Los autores agradecen a Javier Mar Medina por el suministro del material bibliográfico, a Gilma Hernández por sus valiosos aportes y a todos los pacientes que hicieron posibles estos hallazgos.
Financiamiento. Esta investigación se realizó con recursos de la EPS SURA, la Universidad Nacional de Colombia sede Medellín y el Centro Clínico y de Investigación SICOR, Medellín, Colombia. Los patrocinadores no participaron de ninguna manera en el diseño del estudio, recolección y análisis de los datos, ni en la decisión de publicar o presentar el manuscrito.

\section{REFERENCIAS}

1. Yusuf S, Reddy S, Ounpuu S, Anand S. Global burden of cardiovascular diseases: part I: general considerations, the epidemiologic transition, risk factors, and impact of urbanization. Circulation. 2001; 104(22):2746-53.

2. Becker RC, Meade TW, Berger PB, Ezekowitz M, O'Connor CM, Vorchheimer $\mathrm{DA}$, et al. The primary and secondary prevention of coronary artery disease: American College of Chest Physicians Evidence-Based Clinical Practice Guidelines (8a. edición). Chest. 2008; 133(6S):776S-814S.

3. Morrow T. How to implement a disease management program: ten steps to success. Manag Care Interface. 2005; 18(1):50-2.

4. Lorig KR, Ritter P, Stewart AL, Sobel DS, Brown BW, Jr., Bandura A, et al. Chronic disease self-management program: 2-year health status and health care utilization outcomes. Med Care. 2001;39(11):1217-23.

5. Flynn FM, Cafarelli M, Petrakos K, Christophersen P. Improving outcomes for acute coronary syndrome patients in the hospital setting: successful implementation of the American Heart Association "Get With The Guidelines" program by Phase I cardiac rehabilitation nurses. J Cardiovasc Nurs. 2007;22(3):166-76.

6. Jack BW, Chetty VK, Anthony D, Greenwald JL, Sanchez GM, Johnson AE, et al. A reengineered hospital discharge program to decrease rehospitalization: a randomized trial. Ann Intern Med. 2009; 150(3):178-87.

7. Casalino L, Rittenhouse D, Gillies R, Shortell S. Specialist Physician Practices as Patient-Centered Medical Homes. N Engl J Med. 2010;262:1555-15558.

8. Nutting PA, Crabtree BF, Miller WL, Stange KC, Stewart E, Jaen C. Transforming physician practices to patient-centered medical homes: lessons from the national demonstration project. Health Aff (Millwood). 2011;30(3):439-45.

9. Briggs A, Sculpher M. An introduction to markov modelling for economic evaluation. Pharmacoeconomics. 1998;13(4): 397-409.

10. López-Bastida J, Oliva J, Antoñanzas F, García-Altés A, Gisbert R, Mar J, et al. Spanish recommendations on economic evaluation of health technologies. Eur J Health Econ. 2010;11(5):513-20.

11. Aristizábal D, Gallo J, Valencia A, Jaime J, Correa M, Aristizábal A, et al. Effect of a comprehensive ambulatory care model on outcomes of patients with acute coronary syndrome in Colombia. Rev Panam Salud Publica. 2015;38(5):362-9.

12. Paasche-Orlow MK, Riekert KA Bilderback A, Chanmugam A, Hill P, Rand $\mathrm{CS}$, et al. Tailored education may reduce health literacy disparities in asthma selfmanagement. Am J Respir Crit Care Med. 2005;172(8):980-6.

13. Terol E, Hamby EF, Minue S. Gestión de Enfermedades (Disease Management): una aproximación integral a la provisión de cuidados sanitarios. Medifam. 2001; 11:7-20.

14. Aoka Y, Hagiwara N, Kasanuki H. Heterogeneity of hemodynamic parameters in untreated primary hypertension, and individualization of antihypertensive therapy based on noninvasive hemodynamic measurements. Clin Exp Hypertens. 2013;35(1):61-6.

15. Tang WH, Tong W. Measuring impedance in congestive heart failure: current options and clinical applications. Am Heart J. 2009;157(3):402-11.

16. Mancia G, Fagard R, Narkiewicz K, Redon J, Zanchetti A, Bohm M, et al. 2013 ESH/ ESC Guidelines for the management of arterial hypertension: The Task Force for the management of arterial hypertension of the European Society of Hypertension (ESH) and of the European Society of Cardiology (ESC). Eur Heart J. 2013;34(28):2159-219.

17. Montalescot G, Sechtem U, Achenbach S, Andreotti F, Arden C, Budaj A, et al. 2013 ESC guidelines on the management of stable coronary artery disease: The Task nary artery disease of the European Society of Cardiology. Eur Heart J. 2013; 34(38):2949-3003.

18. Resolución 8430 de 1993. Por la cual se establecen las normas científicas, técnicas y administrativas para la investigación en salud. Bogotá: Ministerio de Protección Social; 1993.

19.World Medical A. World Medical Association Declaration of Helsinki: ethical principles for medical research involving human subjects. JAMA. 2013; 310(20):2191-4.

20. Menard S. Coefficients of determination for multiple logistic regression analysis. The American Statistician. 1989;54:17-24.

21. Goldsmith KA, Dyer MT, Schofield PM, Buxton MJ, Sharples LD. Relationship Force on the management of stable coro-

Conflictos de interés. Ninguno declarado por los autores.

Declaración. Las opiniones expresadas en este manuscrito son responsabilidad de los autores y no reflejan necesariamente los criterios ni la política de la RPSP/PJPH o de la OPS.

between the EQ-5D index and measures of clinical outcomes in selected studies of cardiovascular interventions. Health Qual Life Outcomes. 2009;7:96.

22. Duque S, Vásquez J. Riesgo cardiovascular, calidad de vida y años de vida ajustados por calidad: un estudio de caso. Investigación y educación en enfermería. 2010;28(1).

23. Tan-Torres Edejer T, Baltussen R, Adam R, Hutubessy A, Acharya A, Evans DB, et al. Making choices in health: WHO guide to cost-effectiveness analysis. Geneva: World Health Organization; 2003.

24. Briggs A, Sculpher M, Claxton K. Decision modelling for health economic evaluation. Oxford, UK: Oxford University Press; 2006.

25. Mar J, Antoñanzas F, Pradas R, Arrospide A. Los modelos de Markov probabilísticos en la evaluación económica de tecnologías sanitarias: una guía práctica. Gaceta Sanitaria. 2010;24(3):209-14.

26. Oldridge NB, Pakosh MT, Thomas RJ. Cardiac rehabilitation in low- and middle-income countries: a review on cost and cost-effectiveness. International Health. 2016;8(2):77-82.

27. Khawaja FJ, Shah ND, Lennon RJ, Slusser JP, Alkatib AA, Rihal CS, et al. Factors associated with 30 -day readmission rates after percutaneous coronary intervention. Arch Intern Med. 2012;172(2):112-7.

28. Ochoa J, McEwen J, Aristizábal D. Principios de la evaluación hemodinámica no invasiva con cardiografía de impedancia. Revista Colombiana de Cardiología. 2009;16(2):91-102.

29. Smith RD, Levy P, Ferrario CM. Value of noninvasive hemodynamics to achieve blood pressure control in hypertensive subjects. Hypertension. 2006;47(4):771-7.

30. Jorstad HT, von Birgelen C, Alings AM, Liem A, van Dantzig JM, Jaarsma W, et al. Effect of a nurse-coordinated prevention programme on cardiovascular risk after an acute coronary syndrome: main results of the RESPONSE randomised trial. Heart. 2013;99(19):1421-30.

31. Wong WP, Feng J, Pwee KH, Lim J. A systematic review of economic evaluations of cardiac rehabilitation. BMC health services research. 2012;12:243.

32. Yu CM, Lau CP, Chau J, McGhee S, Kong SL, Cheung BM, et al. A short course of cardiac rehabilitation program is highly cost effective in improving longterm quality of life in patients with recent 
myocardial infarction or percutaneous coronary intervention. Archives of physical medicine and rehabilitation. 2004;85(12): 1915-22.

33. Georgiou D, Chen Y, Appadoo S, Belardinelli R, Greene R, Parides MK, et al. Cost-effectiveness analysis of long-term moderate exercise training in chronic heart failure. Am J Cardiol. 2001;87(8): 984-8; a4.

34. Grace SL, Turk-Adawi KI, Contractor A, Atrey A, Campbell N, Derman W, et al. Cardiac rehabilitation delivery model for low-resource settings. Heart. 2016;102(18): 1449-55.

35. Marchionni N, Fattirolli F, Fumagalli S, Oldridge N, Del Lungo F, Morosi L, et al. Improved exercise tolerance and quality of life with cardiac rehabilitation of older patients after myocardial infarction: results of a randomized, controlled trial. Circulation. 2003;107(17):2201-6.

36. Oldridge N, Furlong W, Feeny D, Torrance G, Guyatt G, Crowe J, et al. Economic evaluation of cardiac rehabilitation soon after acute myocardial infarction. Am J Cardiol. 1993;72(2):154-61.
37. Balady GJ, Williams MA, Ades PA, Bittner V, Comoss P, Foody JM, et al. Core components of cardiac rehabilitation/secondary prevention programs: 2007 update: a scientific statement from the American Heart Association Exercise, Cardiac Rehabilitation, and Prevention Committee, the Council on Clinical Cardiology; the Councils on Cardiovascular Nursing, Epidemiology and Prevention, and Nutrition, Physical Activity, and Metabolism; and the American Association of Cardiovascular and Pulmonary Rehabilitation. Circulation. 2007;115(20): 2675-82.

38. Anchique Santos CV, Lopez-Jimenez F, Benaim B, Burdiat G, Fernandez Coronado R, Gonzalez G, et al. Cardiac rehabilitation in Latin America. Progr Cardiovasc Dis. 2014;57(3):268-75.

39. Kuhr EM, Ribeiro RA, Rohde LE, Polanczyk CA. Cost-effectiveness of supervised exercise therapy in heart failure patients. Value Health. 2011;14(S1):S100-7.

40. Rincon M, Rojas MX, Rodriguez Romero VA, Tamayo DC, Franco C, Castro H, et al. Economic Evaluation of Exercise-Based
Cardiac Rehabilitation Programs for Chronic Heart Failure Patients in Colombia. J Cardiopulm Rehabil Prev. 2016;36(1):12-9.

41. López-Montecinos P, Rebolledo J, Gómez J. Costo efectividad de un programa de rehabilitación cardiovascular modelo para personas post infarto agudo al miocardio en el Servicio de Salud Metropolitano Norte. Rev Med Chile 2016;144:456-64.

42. García-Castillo A, Jerjes-Sánchez C, Martínez Bermúdez P, Azpiri-López JR, Autrey Caballero A, Martínez Sánchez C, et al. Registro Mexicano de Síndromes Coronarios Agudos: RENASICA II Mexican Registry of Acute Coronary Syndromes. Archivos de Cardiología de México. 2005;75:6-19.

Manuscrito recibido el 20 de febrero de 2017. Aceptado para publicación, tras revisión, el 19 de junio de 2017.

Objective. To evaluate the cost-effectiveness of an integral model of ambulatory treatment in patients who presented an acute coronary syndrome.

Methods. An economic evaluation was made from a quasi-experimental interven-

Evaluation of the cost-effectiveness of an integral model of ambulatory treatment in patients with acute coronary syndrome: application of a probabilistic Markov model

Keywords tion study, which included 442 patients aged 30 to 70 years who presented an acute coronary syndrome. The intervention group $(n=165)$ received an integral model of ambulatory treatment based on managed care (disease management), while the control group $(n=277)$ received conventional cardiovascular rehabilitation. During one year of follow-up, the presentation of cardiovascular events and hospitalizations was evaluated. A probabilistic Markov model was developed. The study perspective was applied within the General System of Health Social Security in Colombia, including the direct health costs; the time horizon was 50 years with discounts of $3.42 \%$ for costs and effectiveness; and the measure of effectiveness was quality-adjusted life years (QALYs). A probabilistic and multivariate sensitivity analysis was performed using the Montecarlo simulation.

Results. During the year of follow-up, the direct costs related to the value paid were, on average, USD 2577 for the control group and USD 2245 for the intervention group. In the probabilistic sensitivity analysis, $91.3 \%$ of the simulations were located in the quadrant corresponding to incremental negative costs and positive incremental effectiveness (evaluated intervention at a lower cost, more effective). In the simulations, an average annual savings per patient of USD 1215 per QALY was observed.

Conclusions. The integral model of ambulatory treatment implemented in patients who suffered an acute coronary syndrome was found to be less expensive and more effective compared to conventional care. Considering it is a dominant alternative, it is recommended as a model of care in this population.

Cost-effectiveness evaluation; Markov chains; acute coronary syndrome; ambulatory care; disease management; cardiac rehabilitation; Colombia. 
RESUMO

Avaliação da relação custo-eficácia de um modelo integral de tratamento ambulatorial em pacientes com síndrome coronariana aguda: aplicação de um modelo probabilístico de Markov

Palavras-chave
Objetivo. Avaliar a relação custo-eficácia de um modelo integral de tratamento ambulatorial em pacientes que apresentaram síndrome coronariana aguda.

Métodos. Uma avaliação econômica foi feita a partir de um estudo de intervenção quase experimental, que incluiu 442 pacientes com idade entre 30 a 70 anos que apresentaram síndrome coronariana aguda. O grupo de intervenção $(n=165)$ recebeu um modelo integral de tratamento ambulatorial com base em cuidados gerenciados (gerenciamento de doenças), enquanto o grupo controle $(n=277)$ recebeu reabilitação cardiovascular convencional. Durante um ano de acompanhamento, foi avaliada a apresentação de eventos cardiovasculares e hospitalizações. Um modelo probabilístico de Markov foi desenvolvido. A perspectiva do estudo foi aplicada no Sistema Geral de Segurança Social na Saúde da Colômbia, incluindo os custos diretos de saúde; o horizonte temporal foi de 50 anos com descontos de 3,42\% em custos e efetividade; e a medida de eficácia foi os anos de vida ajustados pela qualidade (QALY). Uma análise de sensibilidade probabilística e multivariada foi realizada utilizando a simulação de Montecarlo.

Resultados. Durante o ano de acompanhamento, os custos diretos relacionados ao valor pago foram, em média, USD 2577 para o grupo controle e USD 2245 para o grupo de intervenção. Na análise de sensibilidade probabilística, foram localizadas $91,3 \%$ das simulações no quadrante correspondente a custos negativos incrementais e eficácia incremental positiva (intervenção avaliada a menor custo, mais efetiva). Nas simulações, observou-se uma economia anual média por paciente de US \$ 1215 por QALY.

Conclusões. O modelo integral de tratamento ambulatorial implementado em pacientes que sofreram síndrome coronariana aguda foi considerado menos caro e mais eficaz em comparação com os cuidados convencionais. Por ser uma alternativa dominante, é recomendado como modelo de cuidados nesta população.

Avaliação de custo-efetividade; cadeias de Markov; síndrome coronariana aguda; assistência ambulatorial; gerenciamento clínico; reabilitação cardíaca, Colômbia. 